\title{
Home Visit Program Improves Technique Survival in Peritoneal Dialysis
}

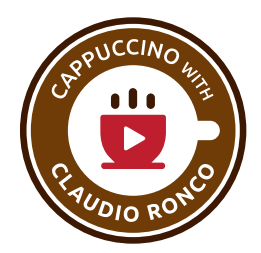

\author{
Francesca Martino $^{a} \quad$ Z. Adıbellib,d G. Mason ${ }^{b} \quad$ A. Nayak ${ }^{b}$, f $\quad$ W. Ariyanon ${ }^{b, g}$ \\ E. Rettore ${ }^{c}$ Carlo Crepaldi $^{a}$ Mariapia Rodighiero ${ }^{a}$ Claudio Ronco $^{a, b}$ \\ ${ }^{a}$ Department of Nephrology, Dialysis, and Kidney Transplant, and ${ }^{b}$ International Renal Research Institute of Vicenza \\ (IRRIV), San Bortolo Hospital, Vicenza, and ' Department of Statistical Sciences, University of Padova, Padova, Italy; \\ ${ }^{\mathrm{d}}$ Nephrology Department, Antalya Ataturk State Hospital, Antalya Turkey; Departments of e Chemical Engineering \\ and ${ }^{\mathrm{f}}$ Economics, BITS, Pilani, India; ${ }^{9}$ Cardiometabolic Center, BNH Hospital, Bangkok, Thailand
}

\section{Key Words}

Home visit · Technique survival · Peritoneal dialysis

\begin{abstract}
Background: Peritoneal dialysis (PD) is a home therapy, and technique survival is related to the adherence to PD prescription at home. The presence of a home visit program could improve PD outcomes. We evaluated its effects on clinical outcome during 1 year of follow-up. Methods: This was a case-control study. The case group included all 96 patients who performed PD in our center on January 1, 2013, and who attended a home visit program; the control group included all 92 patients who performed PD on January 1, 2008. The home visit program consisted of several additional visits to reinforce patients' confidence in PD management in their own environment. Outcomes were defined as technique failure, peritonitis episode, and hospitalization. Clinical and dialysis features were evaluated for each patient. $\boldsymbol{R e}$ sults: The case group was significantly older $(p=0.048)$, with a lower grade of autonomy ( $p=0.033)$, but a better hemoglobin level $(p=0.02)$ than the control group. During the observational period, we had 11 episodes of technique failure. We found a significant reduction in the rate of technique failure in the case group $(p=0.004)$. Furthermore, survival
\end{abstract}

analysis showed a significant extension of PD treatment in the patients supported by the home visit program (52 vs. 48.8 weeks, $p=0.018$ ). We did not find any difference between the two groups in terms of peritonitis and hospitalization rate; however, trends toward a reduction of Gram-positive peritonitis rates as well as prevalence and duration of hospitalization related to PD problems were identified in the case group. The retrospective nature of the analysis was a limitation of this study. Conclusion: The home visit program improves the survival of PD patients and could reduce the rate of Gram-positive peritonitis and hospitalization.

Video Journal Club "Cappuccino with Claudio Ronco" at http://www.karger.com/?doi=365168.

(c) 2014 S. Karger AG, Basel

\section{Introduction}

Peritoneal dialysis (PD) and hemodialysis (HD) are recognized as equivalent dialysis modalities in end-stage renal disease (ESRD). HD, however, is more frequently adopted than PD, especially in Western countries [1]. The higher prevalence of HD is mainly driven by logistic and structural barriers, and is unrelated to better clinical outcomes, cost, and quality of life [2]. Despite the evidence

\section{KARGER}

E-Mail karger@karger.com

www.karger.com/bpu
(C) 2014 S. Karger AG, Basel

0253-5068/14/0374-0286\$39.50/0
Dr. Francesca Martino

Department of Nephrology, Dialysis, and Kidney Transplant San Bortolo Hospital

Viale Rodolfi 37, IT-36100 Vicenza (Italy)

E-Mail francesca.martino.k@gmail.com 
in favor of PD, there is still a growing concern about PD technique failure [3], which is estimated to happen in about $40 \%$ of patients in the first year of PD [4] and is usually caused by recurrent or refractory peritonitis, ultrafiltration failure, and inadequate dialysis. Among the possible strategies to cut technique failure, a good option could be an increase of support for PD patients by means of a home visit program. This could improve the standard of care in many different ways such as optimization of the environment where PD is done, increase in the adherence to the prescription, correction of potential or real hazards that may be related to a risk of infection, and reinforcement of patients' confidence in self-care. Furthermore, home visits seem to contribute to an amelioration of other outcomes such as reduction of peritonitis rates [5-8] and PD-related hospitalizations [8].

Currently, most of the studies on home visit programs have reported mainly the effect on patient compliance $[9$, $10]$ and peritonitis rates [5-8], but no study has evaluated the impact of a home visit program on PD technique failure. The aim of the present study is to examine the effect of a regular a home visit program on clinical outcomes such as technique survival, peritonitis, and hospitalization rates during 1 year of follow-up.

\section{Subjects and Methods}

\section{Study Design}

We conducted a retrospective case-control study about the effect of a regular home visit protocol in a Vicenza PD center. The case group consisted of all the patients who attempted PD in our center on January 1,2013, and who were supported by home visits, while the control group included all the patients who attempted PD at our center on January 1, 2008. The patients who were dialyzed in our center on both dates were excluded. All patients were followed for 1 year. The observational period was between January 1, 2013, and December 31, 2013, for the case group, and between January 1, 2008, and December 31, 2008, for the control group.

\section{Home Visit Program}

The Vicenza home visit program started in autumn of 2011 and was entrusted to nurses skilled in PD features. Every patient who participated in the program received supplemental home visits by nurses. The home visits were scheduled every 3 months between two visits in the PD center. Furthermore, an additional home visit was administered on the base of medical suggestions.

During the home visit, the nurse supervised the environment where the patient had the PD exchange, possible mistakes during the procedures, the place where the PD material was stored and conditions of the storage, and compliance to pharmacological and dialysis therapy. Finally, the nurse supported patients by suggesting possible solutions, reinforcing patient knowledge, and/or anticipating a medical visit to the PD center.
Table 1. Baseline characteristics of all patients in the case and the control group

\begin{tabular}{|c|c|c|c|}
\hline Variable & $\begin{array}{l}\text { Case group } \\
(\mathrm{n}=96)\end{array}$ & $\begin{array}{l}\text { Control group } \\
(\mathrm{n}=92)\end{array}$ & $\mathrm{p}$ \\
\hline Men & 63.5 & 64.1 & $0.933^{1}$ \\
\hline Diabetes & 31.2 & 24.4 & $0.301^{1}$ \\
\hline Caregivers & 37 & 39.5 & $0.723^{1}$ \\
\hline $\mathrm{PD}$ vintage classes & & & $0.21^{1}$ \\
\hline$<6$ months & 21.9 & 23.9 & \\
\hline $6-24$ months & 37.5 & 22.8 & \\
\hline$>24$ months & 40.6 & 53.3 & \\
\hline Age, years & $65.44 \pm 14.12$ & $61.52 \pm 13.501$ & $0.048^{2}$ \\
\hline $\mathrm{BMI}$ & $27.48 \pm 5.5$ & $27.9 \pm 5.5$ & $0.59^{2}$ \\
\hline Hemoglobin, g/dl & $11.6 \pm 5.3$ & $11.1 \pm 1.9$ & $0.02^{2}$ \\
\hline Albumin, g/dl & $3.6(3.2-3.9)$ & $3.7(3.4-3.9)$ & $0.393^{3}$ \\
\hline $\mathrm{Kt} / \mathrm{V}$ & $1.9(1.75-2.38)$ & $1.97(1.78-2.17)$ & $0.961^{3}$ \\
\hline Karnofsky score & $80(60-100)$ & $90(70-100)$ & $0.033^{3}$ \\
\hline
\end{tabular}

Values represent percentages, means \pm SD or medians (interquartile range). ${ }^{1}$ Pearson's $\chi^{2}$ test. ${ }^{2}$ t test with equal variances assumed. ${ }^{3}$ Mann-Whitney U test.

\section{Clinical Evaluation}

Baseline demographic and clinical data were recorded at the beginning of 2014 for both groups on the basis of clinical charts. In the observational period, we evaluated the presence of several clinical outcomes such as removal of peritoneal catheter, shift to $\mathrm{HD}$, occurrence of kidney transplant, peritonitis episode, and hospitalization in our ward. Moreover, we collected the following clinical features and blood examination at the start of the observational period: age, vintage of renal replacement therapy, Karnofsky score, type of $\mathrm{PD}$, presence of diabetes, BMI, albumin, hemoglobin, and Kt/V urea.

\section{Definition of Outcomes}

PD technique failure was defined as a switch to HD for a period longer than 60 consecutive days. Furthermore, we analyzed the causes of PD failure such as recurrent or refractory peritonitis, fluids adequacy imbalance, and inadequate dialysis index.

Peritonitis was defined according to the criteria of the International Peritoneal Dialysis Society guidelines [11]. Specifically, in the first episode of peritonitis we evaluated the duration of antibiotic therapy and the type of peritonitis according peritoneal effluent culture.

Admission to the nephrology ward was related to the PD problem. Specifically, we assessed the number and the duration of hospitalizations.

\section{Control Selection}

The selection of the control group was applied on the basis of several considerations such as the need to obtain the highest possible number of patients, need to have similar support and similar treatment in both groups, and opportunity to simply find medical records. We chose the patients from 2008 because they better met these criteria. Specifically, the protocols related to the methodol- 
Table 2. Outcomes in the case and the control group

\begin{tabular}{|c|c|c|c|}
\hline Outcome & Case group $(n=96)$ & Control group $(n=92)$ & $\mathrm{p}$ \\
\hline \multicolumn{4}{|l|}{ PD technique failure } \\
\hline PD failure & 11.5 & 23.3 & $0.004^{1}$ \\
\hline Recurrent or refractory peritonitis & 0 & 4.3 & $0.089^{1}$ \\
\hline Fluids adequacy imbalance & 1.6 & 2.2 & \\
\hline Inadequate dialysis & 0 & 2.2 & \\
\hline Other causes & 0 & 1.1 & \\
\hline \multicolumn{4}{|l|}{ Peritonitis episode } \\
\hline One peritonitis episode & 29.2 & 28.3 & $0.65^{1}$ \\
\hline Gram-positive & 13.5 & 18.5 & $0.14^{1}$ \\
\hline Gram-negative & 9.4 & 6.5 & \\
\hline Sterile & 6.3 & 5.4 & \\
\hline Antibiotic length, days & $10(10-14.5)$ & $13.5(10-19.75)$ & $0.19^{2}$ \\
\hline Two or more peritonitis episodes & 9.4 & 2.2 & $0.54^{1}$ \\
\hline \multicolumn{4}{|l|}{ Hospitalization event } \\
\hline Admission to the nephrology ward & 13.5 & 15.2 & $0.743^{1}$ \\
\hline Related to PD therapy & 14.6 & 21.7 & $0.203^{1}$ \\
\hline Hospitalization length, days & $14(9.75-23.5)$ & $10.5(5.5-19.75)$ & $0.20^{2}$ \\
\hline
\end{tabular}

Values represent percentages or medians (interquartile range). ${ }^{1}$ Pearson's $\chi^{2}$ test. ${ }^{2}$ Mann-Whitney U test.

ogy of PD exchange and exit-site medication were not modified; clinical notices and blood examinations were reported with the same medical records software in a standardized manner.

\section{Statistical Analysis}

All continuous variables were represented as means \pm SD or as median values with interquartile range according to their distribution. Categorical variables were reported as percentage of cases. Normality of variable distribution was tested by a Shapiro-Wilk W test; a t test, Mann-Whitney U test, and Pearson's $\chi^{2}$ test were used to compare continuous and categorical variables, as appropriate. Kaplan-Meier curves were used to report the PD failure in case and control groups. Univariate Cox regression models addressed time to PD failure. All reported p values were two sided, and statistical significance was set at $\mathrm{p}<0.05$. Statistical analysis was performed with SPSS version 19.0 (SPSS Inc., Chicago, Ill., USA).

\section{Results}

We evaluated 188 patients, 96 in the case group and 92 in the control group. Table 1 shows the baseline characteristics for each group and the salient difference between the control and case groups. Specifically, a higher level of hemoglobin and older age were found in the case group, while higher Karnofsky scores were found in the control group.

Eleven patients experienced technique failure during observational periods. Furthermore, prevalence of tech-

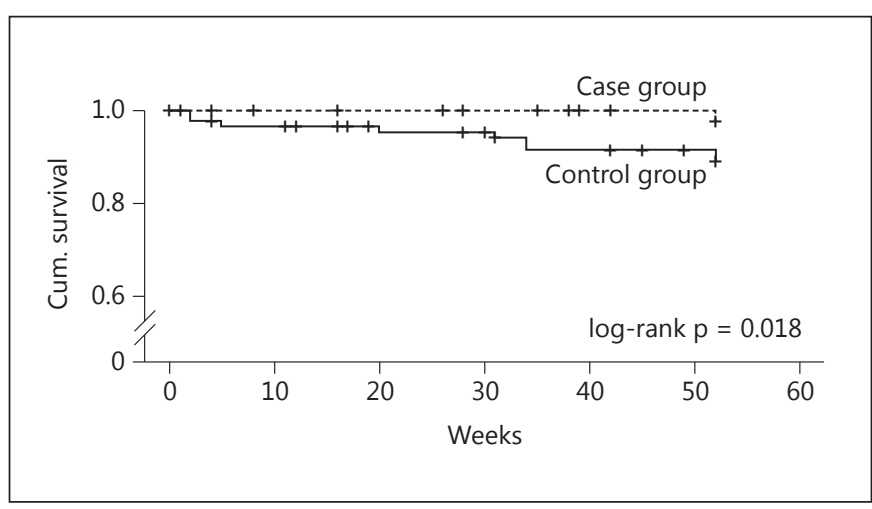

Fig. 1. Survival functions. Kaplan-Meier curves of PD technique failure recurrence in the case and the control group.

nique failure was significantly lower in the case group $(\mathrm{p}=0.004)$. All details about the causes of technical failure are reported in table 2. Moreover, survival analysis showed a significantly longer extension of PD treatment in the patients supported by the home visit program ( 52 vs. 48.8 weeks, respectively; $\mathrm{p}=0.018$; fig. 1 ). Table 3 summarizes analysis for the prediction of PD technique failure. In univariate analysis, the presence of a home visit program predicted PD technique survival. 
Table 3. Univariate Cox regression addressed to PD technical failure

\begin{tabular}{llcl}
\hline Covariate & HR & $95 \% \mathrm{CI}$ & $\mathrm{p}$ \\
\hline Home visit program & 0.192 & $0.041-0.889$ & 0.035 \\
Age & 1.002 & $0.94-1.06$ & 0.94 \\
Diabetes & 1.509 & $0.377-6.040$ & 0.661 \\
Karnofsky score $<80$ & 1.362 & $0.41-4.46$ & 0.61 \\
Hemoglobin & 0.79 & $0.585-1.066$ & 0.122 \\
Albumin & 0.575 & $0.248-1.336$ & 0.198 \\
Kt/V & 0.281 & $0.041-1.928$ & 0.407
\end{tabular}

During observational periods, 59 patients had at least 1 episode of peritonitis. No significant difference was observed in the two groups in terms of both first $(\mathrm{p}=0.65)$ and multiple peritonitis episodes $(\mathrm{p}=0.54)$. The overall peritonitis rate was 1 episode every 35 months in the control group and 1 episode every 30 months in the case group. Moreover, we found an interesting reduction of Gram-positive peritonitis and antibiotic therapy duration in the home visit program (table 2).

Finally, we had 32 hospitalizations in the nephrology ward during the two periods of observation. We did not see any difference in the number of admissions in the nephrology ward for the two groups $(\mathrm{p}=0.74)$, but we noticed a little reduction of hospitalization and of hospitalization length related to PD complications in the case group (table 2).

\section{Discussion}

In the present study, we analyzed the effect of a wellstructured home visit program in PD patients at San Bortolo Hospital in Vicenza, Italy. We observed that a significant reduction in PD technique failure was associated with a significant prolongation of PD treatment in the patients who participated in the home visit program. Currently, no study about home visit programs has been performed to assess PD technique failure. Some studies have described home visit programs, but evaluated mainly patient compliance [12] and patients' environments and skills $[10,13,14]$; however, they did not consider the effect of such programs on preventing or delaying the shift to HD. In this regard, our results seem to be the missing link between the effort of home visit programs and the achievement of an incisive outcome like the prevention of PD technique failure. The prolongation of PD seems to be the positive effect of some factors strictly related with home visit programs such as improvement of patient knowledge about PD features, continuous retraining, discovery of real compliance in dialysis and pharmacological treatment, awareness of patient's environment, and possible psychological effects. Finally, improved technique survival on PD may provide benefits in terms of the cost of providing care to ESRD patients [15]. Some studies have shown lower healthcare costs in patients who received only PD therapy as renal replacement therapy $[16$, 17] in Western countries. Consequently, keeping a patient on PD for a longer period could be cost-effective. Considering the growing number of ESRD patients and the need to supply the increasing requirement of renal replacement therapy, it does not seem pointless to evaluate the possible strategy to comply with this need.

Surprisingly, peritonitis rates in our series did not show a significant fall with the home visit program, although we did observe a positive impact on the incidence of Gram-positive peritonitis and on the duration of antibiotic therapy. To date, there have only been a few studies which have analyzed the effect of home visits on peritonitis rates, and just one study that has observed the effect of patient's knowledge on peritonitis rate. All these studies have shown improvement in peritonitis rates with home visit programs [5-7], while our results did not achieve this outcome. This discordancy could be explained by the different ages and the different grades of autonomy of our patients in the home visit and control groups. The control patients were significantly younger and more autonomous, and these conditions could dilute the advantage related with the home visit program. In this regard, Nessim et al. [18] demonstrated that age was an independent predictor of peritonitis events, whereas some studies have shown a higher vulnerability to infection in the patients who had lower Karnofsky scores [19$21]$. On the other hand, we observed a trend toward a reduction of Gram-positive peritonitis and an increase of Gram-negative peritonitis. Specifically, Gram-negative peritonitis is frequently associated with some conditions such as bowel disease and transmural migration [22], which can hardly be corrected by an educational program. Furthermore, Gram-negative peritonitis is more likely to cause PD technique failure [23]. Therefore, home visit programs could have a better impact on the primary outcome than we observed in our series.

This study is important because it shows a positive effect of home visit programs, with a significant reduction in PD technique failure. Based on this finding, we consider the presence of home visit programs as a crucial el- 
ement in the care of PD patients. It allows the opportunity to improve the quality of care and humanize renal replacement therapy, as well as the possibility to reduce the costs in the management of ESRD patients through the prolongation of PD [15].

The present study is not devoid of limitations. The retrospective study design is the most relevant because it reduced our likelihood to evaluate possible confounding factors such as the different age and autonomy of patients. Moreover, cost analysis is not available at the present moment.
In conclusion, the presence of a home visit program leads to a prolongation of PD therapy and seems to reduce the rate of Gram-positive peritonitis and hospitalization.

\section{Disclosure Statement}

The study did not receive any kind of funding. Prof. C. Ronco has received speaking honoraria from Abbott, Alere, and Gambro. All other authors have no competing interests.

\section{References}

1 Farrington K, Rao R, Stenkamp R, Ansell D, Feest T: All patients receiving renal replacement therapy in the United Kingdom in 2005 (chapter 4). Nephrol Dial Transplant 2007; 22(Suppl 7):vii30-vii50.

-2 Ghaffari A, Kalantar-Zadeh K, Lee J, Maddux F, Moran J, Nissenson A: PD First: peritoneal dialysis as the default transition to dialysis therapy. Semin Dial 2013;26:706-713.

3 Mendelssohn DC, Langlois N, Blake PG: Peritoneal dialysis in Ontario: a natural experiment in physician reimbursement methodology. Perit Dial Int 2004;24:531-537.

4 Chidambaram M, Bargman JM, Quinn RR, Austin PC, Hux JE, Laupacis A: Patient and physician predictors of peritoneal dialysis technique failure: a population based, retrospective cohort study. Perit Dial Int 2011;31: 565-573.

5 Ellis EN, Blaszak C, Wright S, Van Lierop A: Effectiveness of home visits to pediatric peritoneal dialysis patients. Perit Dial Int 2012;32: 419-423.

6 Bordin G, Casati M, Sicolo N, Zuccherato N, Eduati V: Patient education in peritoneal dialysis: an observational study in Italy. J Ren Care 2007;33:165-171.

7 Verger C, Duman M, Durand PY, Veniez G, Fabre E, Ryckelynck JP: Influence of autonomy and type of home assistance on the prevention of peritonitis in assisted automated peritoneal dialysis patients. An analysis of data from the French Language Peritoneal Dialysis Registry. Nephrol Dial Transplant 2007; 22:1218-1223.
Sayed SA, Abu-Aisha H, Ahmed ME, Elamin $S$ : Effect of the patient's knowledge on peritonitis rates in peritoneal dialysis. Perit Dial Int 2013;33:362-366.

-9 Bernardini J, Piraino B: Compliance in CAPD and CCPD patients as measured by supply inventories during home visits. Am J Kidney Dis 1998;31:101-107.

10 Ozturk S, Yucel L, Guvenc S, Ekiz S, Kazancioglu R: Assessing and training patients on peritoneal dialysis in their own homes can influence better practice. J Ren Care 2009;35: 141-146.

11 Li PK, Szeto CC, Piraino B, et al: Peritoneal dialysis-related infections recommendations: 2010 update. Perit Dial Int 2010;30:393-423.

12 Bernardini J, Piraino B: Measuring compliance with prescribed exchanges in CAPD and CCPD patients. Perit Dial Int 1997;17:338342.

13 Russo R, Manili L, Tiraboschi G, et al: Patient re-training in peritoneal dialysis: why and when it is needed. Kidney Int Suppl 2006; 103:S127-S132.

14 Kazancioglu R, Ozturk S, Ekiz S, Yucel L, Dogan S: Can using a questionnaire for assessment of home visits to peritoneal dialysis patients make a difference to the treatment outcome? J Ren Care 2008;34:59-63.

15 Chui BK, Manns B, Pannu N, et al: Health care costs of peritoneal dialysis technique failure and dialysis modality switching. Am J Kidney Dis 2013;61:104-111.

16 Sennfalt K, Magnusson M, Carlsson P: Comparison of hemodialysis and peritoneal dialysis - a cost-utility analysis. Perit Dial Int 2002; 22:39-47.
17 Baboolal K, McEwan P, Sondhi S, Spiewanowski P, Wechowski J, Wilson K: The cost of renal dialysis in a UK setting - a multicentre study. Nephrol Dial Transplant 2008; 23:1982-1989.

18 Nessim SJ, Bargman JM, Austin PC, Nisenbaum R, Jassal SV: Predictors of peritonitis in patients on peritoneal dialysis: results of a large, prospective Canadian database. Clin J Am Soc Nephrol 2009;4:1195-1200.

19 Cardoso T, Teixeira-Pinto A, Rodrigues PP, Aragao I, Costa-Pereira A, Sarmento AE: Predisposition, insult/infection, response and organ dysfunction (PIRO): a pilot clinical staging system for hospital mortality in patients with infection. PloS One 2013;8:e70806

20 Salami AK, Desalu OO, Adeoye PO, Akanbi Ii AA, Oguntoyinbo AE, Fadeyi A: Influence of performance status on the risk and outcome of nosocomial pneumonia in the elderly admitted to an open medical ward. West Afr J Med 2010;29:332-338.

21 Sakuma Y, Katoh T, Owada K, et al: Initial functional status predicts infections during steroid therapy for renal diseases. Clin Nephrol 2005;63:68-73.

22 Szeto CC, Chow KM: Gram-negative peritonitis - the Achilles heel of peritoneal dialysis? Perit Dial Int 2007;27(Suppl 2):S267-S271.

23 Bunke CM, Brier ME, Golper TA: Outcomes of single organism peritonitis in peritoneal dialysis: Gram negatives versus Gram positives in the Network 9 Peritonitis Study. Kidney Int 1997;52:524-529. 